\title{
Role of spindle pole body component 25 in neurodegeneration
}

\author{
Feilun Cui ${ }^{1}$, Zhipeng $\mathrm{Xu}^{1}$, Yumei $\mathrm{Lv}^{2}$, Jianpeng $\mathrm{Hu}^{1}$ \\ ${ }^{1}$ Department of Urology, Affiliated People's Hospital of Jiangsu University, Zhenjiang, China; ${ }^{2}$ Department of Health Management Section, \\ Zhenjiang College, Zhenjiang, China \\ Contributions: (I) Conception and design: F Cui, J Hu; (II) Administrative support: F Cui, J Hu; (III) Provision of study materials or patients: F Cui, J \\ $\mathrm{Hu}$; (IV) Collection and assembly of data: All authors; (V) Data analysis and interpretation: F Cui, J Hu; (VI) Manuscript writing: All authors; (VII) \\ Final approval of manuscript: All authors. \\ Correspondence to: Jianpeng Hu. Department of Urology, Affiliated People's Hospital of Jiangsu University, 8 Electric Power Road, Zhenjiang 212001, \\ China. Email: y_t_2016@163.com; Yumei Lv. Department of Health Management Section, Zhenjiang College, 518 Changxiang West Avenue, \\ Zhenjiang 212028, China. Email: 1095085689@qq.com.
}

\begin{abstract}
Background: Aberrant growth and polarization of microglia are critical for pathological initiation and progression of neurodegenerative conditions like Alzheimer's disease (AD). However, the molecular signals that govern the outgrowth of microglia have not yet been fully determined. Spindle pole body component 25 (SPC25) is an important part for forming NDC80 complex, which plays a key role in the assembly of the microtubule-binding domain of kinetochores. Nevertheless, the role of SPC25 in microglial growth during neurodegeneration has not been described before, and was thus addressed in the current study.
\end{abstract}

Methods: We generated an adeno-associated virus (AAV) serotype PHP.B carrying short hairpin RNA (shRNA) for SPC25 (shSPC25) under a microglia-specific TMEM119 promoter (AAV-pTMEM-shSPC25). Serotype PHP.B allowed the virus to cross blood-brain barrier, while TMEM119 promoter allowed specific targeting microglia in vitro and in vivo. We intravenously administrated AAV-pTMEM-shSPC25 to ADprone APP/PS1 male and female mice and determined this effect on microglia proliferation and mouse behavior.

Results: Depletion of SPC25 did not alter polarization of microglia cell polarization in vitro. On the other hand, AD-prone APP/PS1 mice that had received AAV-pTMEM-shSPC25 significantly decreased SPC25 levels in microglia and attenuated microglia proliferation, resulting in significant improvement of the performance of the mice in behavior tests.

Conclusions: Specific depletion of SPC25 in microglia may prevent AD development through suppression of microglia outgrowth. SPC25 may be a promising novel target for preventing AD through microglia.

Keywords: Neurodegeneration; Alzheimer's disease (AD); spindle pole body component 25 (SPC25); microglia

Submitted Jul 09, 2021. Accepted for publication Sep 03, 2021.

doi: 10.21037/atm-21-4064

View this article at: https://dx.doi.org/10.21037/atm-21-4064

\section{Introduction}

Atrophy of brain tissue with loss of neurons and synapses, deposit of extracellular amyloid-beta (A $\beta)$, and intracellular formation of aggregated phosphorylated tau (p-Tau) are hallmarks of Alzheimer's disease (AD), in which the patients experience cognitive and behavioral impairments (1).

Accumulating data have demonstrated that diverse environmental factors may profoundly impact the neurological functionality through modulating the immune system (2). Microglia are the primary immune and cytokineproducing cells of the central nervous system (CNS) (2). Moreover, impairment in microglia can adversely impact brain connectivity and behavior (1). Microglia play a pivotal role in brain development, physiological homeostasis, and dynamic responses under various pathological circumstances, including neurodegenerative diseases like 
$\mathrm{AD}$ (2). Recent evidence has shown that the majority of $\mathrm{AD}$ risk loci are located in or near microglia-specific genes (3-5). Moreover, aberrant growth and polarization of microglia are critical for pathological alteration in $\mathrm{AD}$ (3-5). However, the molecular signals that govern the outgrowth of microglia have not been fully determined.

Spindle pole body component 25 (SPC25) is an important part for forming NDC80 complex, which plays a key role in the assembly of the microtubule-binding domain of kinetochores, which mediate chromosome alignment to the metaphase plate (6). The NDC80 complex has 4 components, including NDC80 (Hec1 in humans), NUF2, spindle pole body component 25 (SPC25), and SPC24 (7). We have previously shown that SPC25 plays a non-redundant role in the proliferation and cell cycle progression of prostate cancer (8), and is the key factor to determine the stemness of prostate cancer cells (9). Most interestingly, a recent study analyzed microglia profiling at single-cell level and showed that microglia significantly upregulated SPC25 (10). However, the exact role of SPC25 in microglia growth during neurodegeneration has not been described before.

We generated an adeno-associated virus (AAV) serotype PHP.B carrying short hairpin RNA (shRNA) for SPC25 (shSPC25) under a microglia-specific TMEM119 promoter (AAV-pTMEM-shSPC25). Serotype PHP.B enabled the virus to cross the blood-brain barrier (BBB), while TMEM119 promoter allowed specific targeting microglia in vitro and in vivo. Following intravenous administration of AAV-pTMEM-shSPC25 to AD-prone APP/PS1 mice, the SPC25 levels in microglia were significantly decreased and microglia proliferation was attenuated, which led to significant improvement of the performance of the mice in behavior tests. Thus, our data suggest that specific depletion of SPC25 in microglia may prevent AD development through suppression of microglia outgrowth. We present the following article in accordance with the ARRIVE reporting checklist (available at https://dx.doi.org/10.21037/ atm-21-4064).

\section{Methods}

\section{Experimental and animal protocols}

Experiments were performed under a project license (SQK-20160037-Y) granted by institutional ethics board of the Affiliated People's Hospital of Jiangsu University, in compliance with institutional guidelines for the care and use of animals. A protocol was prepared before the study without registration. We purchased APPswe/PSEN1dE9 (APP/PS1) (11) transgenic mice from Jackson Labs (Bar Harbor, ME, USA). The mice were individually housed under a $12 \mathrm{~h}$ light-dark cycle (temperature: $23 \pm 3{ }^{\circ} \mathrm{C}$; humidity: $45 \% \pm 5 \%$ ) with ad libitum access to water and food. Both male and female mice were used and distributed evenly in each experimental group. Mice at 3 months of age received viral treatment and were kept for another 3 months to monitor $\mathrm{AD}$ development. For each experiment, power calculations $(\mathrm{P}<0.05)$ were performed to include sufficient numbers of animals to be confident that the observed effects were legitimate. An allocation concealment method was used to provide randomization in allocating experimental units to control and treatment groups. The use of inbred littermate mice in a specified experiment ensured that the potential confounders were minimized. No criteria were used for excluding animals (or experimental units) during the experiment, and no data were excluded during the analysis. The study did not have humane endpoints.

\section{Cell lines}

Several cell lines were used in the current study: HMC3 is a mouse microglia cell line, RAW264.7 is mouse macrophage cell line, $\mathrm{HCN}-2$ is a mouse neuronal cell line, and 3T3 is mouse fibroblast cell line. These cell lines were all purchased from American Type Culture Collection (ATCC, Rockville, MD, USA) and cultured in Dulbecco's modified Eagle medium (DMEM, Gibco; Life Technologies, Carlsbad, CA, USA) with $10 \%$ fetal bovine serum (FBS, Sigma-Aldrich, St Louis, MO, USA), penicillin $(100 \mu \mathrm{g} / \mathrm{mL})$ and streptomycin $(250 \mathrm{ng} / \mathrm{mL})$ at $37{ }^{\circ} \mathrm{C}$, in a $5 \% \mathrm{CO}_{2}$ atmosphere.

\section{Adeno-associated virus design, production and administration}

The sequence for shRNA for SPC25 (shSPC25) is 3 '-CC GGCCATCAAAGCATTTGCAGAAACTCGAG TTTCTGCAAATGCTTTGATGGTTTTTG-5 ', as described (9). The scramble sequence is 5 -GGCTZTT TCGATTTCCCCT-3'. A microglia-specific TMEM119 promoter was purchased from GeneCopoeia (\#34837, GeneCopoeia, Rockville, MD, USA). Plasmid pUCminiiCAP-PHP.eB (Addgene \#103005, Addgene, Watertown, MA, USA) was used for generating adeno-associated virus (AAV) serotype PHP.B vector. The AAV serotype PHP.B 
carrying shSPC25 or scramble under TMEM119 promoter (AAV-pTMEM-shSPC25 or AAV-pTMEM-scramble) also drove a red fluorescent protein (RFP) reporter for tracing the transduced cells. Transfection was done with Lipofectamine 3000 reagent (Invitrogen, Carlsbad, CA, USA). according to the manufacturer's instructions. For tail vein injection, a single injection with $10^{12}$ viral particles in $100 \mu \mathrm{L}$ total volume was performed through the mice tails.

\section{Behavioral and pain assessments}

A Morris water-maze test was used to assess spatial reference memory of the mice. Briefly, all mice were trained in the testing pool with 20 seconds of water exposure daily for the $10 \mathrm{~d}$ immediately before the test. For the test, mice underwent visible platform training for 18 trials in $2 \mathrm{~d}$, and then hidden platform training for another 18 trials in the subsequent $3 \mathrm{~d}$. The mean target quadrant occupancy was recorded. A motor assessment was conducted to assess balance and coordination ability. A beam crossing test was used to assess the movement accuracy.

\section{Quantitative reverse transcription polymerase chain reaction}

We performed RNA extraction and circular DNA (cDNA) synthesis routinely.

Quantitative reverse transcription polymerase chain reaction (RT-qPCR) primers were all purchased from Qiagen (Hilden, Germany). Values were normalized sequentially against $\beta$-actin and experimental controls.

\section{Flow cytometry}

A FlowJo software (Flowjo LLC, Ashland, OR, USA) was used to analyze the flow cytometry data. Macrophages were sorted by an allophycocyanin (APC)-conjugated CD11b and a FITC-conjugated CD45 antibodies [Becton, Dickinson and Company (BD) Biosciences, Shanghai, China].

\section{Enzyme-linked immunosorbent assay, histology, and immunobistochemistry}

Mouse hippocampi were formalin fixed and embedded in paraffin. Hematoxylin counterstaining was performed at the end. used to provide cytologic detail. Standard procedures of peroxidase-attached streptavidin and 3,3'-diaminobenzidine $(\mathrm{DAB})$ as a substrate were sequentially used. Direct fluorescence was used to detect RFP. Immunostaining was done using rabbit anti-SPC25 antibody (Abcam, Dallas, TX, USA), rabbit anti-IBA-1 antibody (Abcam), rabbit anti-A $\beta$ antibody (EMD Millipore, Billerica, MA, USA), or goat anti-phosphorylated Tau (p-Tau) (Santa Cruz Biotechnology, Dallas, TX, USA) or rabbit anti-PHF1 antibody (Abcam) antibodies. For quantitating neuronal cell loss, PHF-1+ neuronal positive cells were analyzed throughout the CA1 region of the hippocampus. Enzymelinked immunosorbent assay (ELISA) for $\mathrm{A} \beta$ and $\mathrm{p}$ Tau was performed using specific kits (R\&D System, Los Angeles, CA, USA) as instructed.

\section{Statistics}

Statistical analysis for all experiments was performed using GraphPad Prism 7 (GraphPad Software, San Diego, CA, USA). All analyses were performed using one-way analysis of variance (ANOVA) with a Bonferroni correction, followed by Fisher's exact test where necessary. Data were presented as mean \pm standard deviation (SD) from at least 5 individuals and were considered significant when $\mathrm{P}<0.05$.

\section{Results}

\section{Generation of AAV-pTMEM-shSPC25 capable of crossing $B B B$}

A recent study to examine microglia profiling at singlecell level showed that microglia significantly upregulated SPC25 (10). However, the exact role of SPC25 in microglia growth during neurodegeneration has not been described before. In order to address this question, we prepared an AAV vector system that specifically depletes SPC25 in microglia. Thus, we chose a specific serotype, PHP. $\mathrm{B}$, which allows the virus in the circulation to cross $\mathrm{BBB}$ (12-14), so that the AAVs could be administered via the tail vein of mice. Moreover, we used a microglia-specific TMEM119 promoter to drive the shSPC25 or scramble sequence (15). With these 2 strategies, we generated AAVpTMEM-shSPC25 and a control AAV-pTMEM-scramble, serotype PHP.B (Figure 1A). The viruses also contained an RFP reporter to allow tracing of the transduced cells (Figure 1A). First, we examined the specificity of these AAVs for microglia. It was found that AAV-pTMEM-scramble transduced mouse microglia cell line HMC3, but not mouse neuronal cell line $\mathrm{HCN}-2$, or mouse macrophage cell line RAW264.7, or mouse fibroblast cell line 3T3, according to 

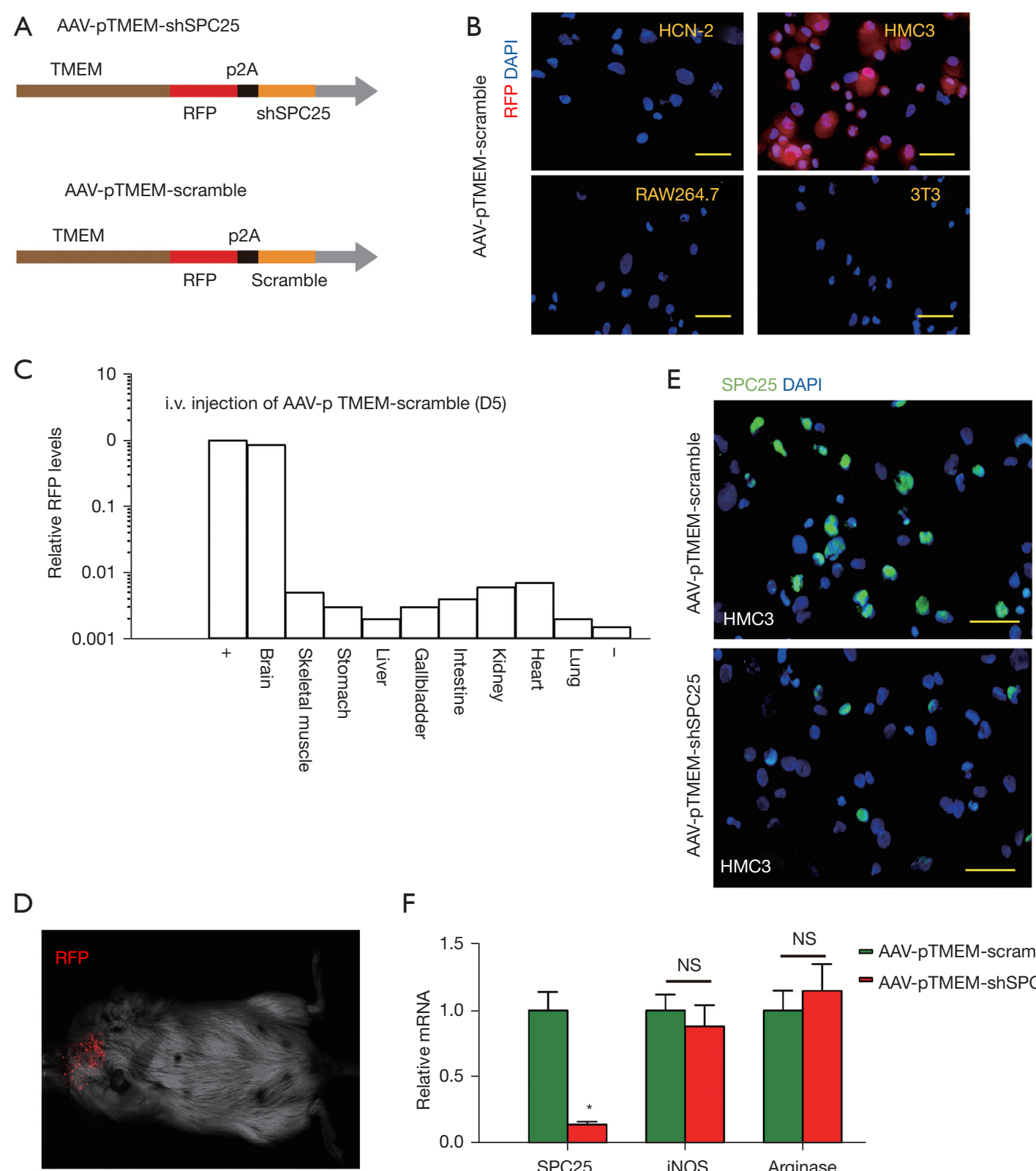

$\mathrm{F}$

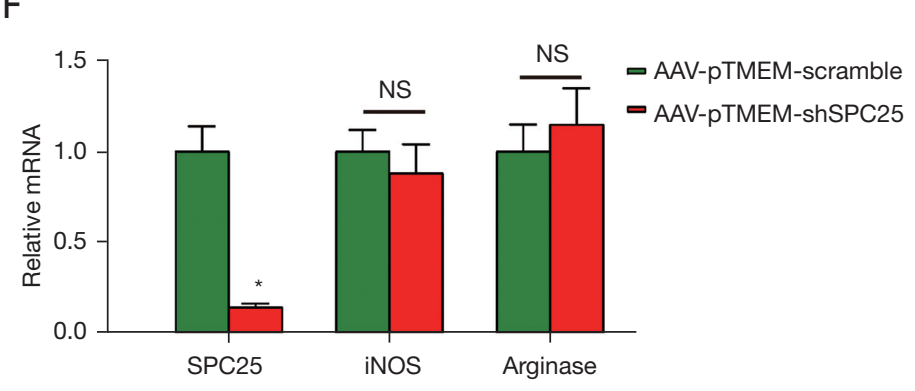

AAV-pTMEM-scramble (D5)

Figure 1 Generation of AAV-pTMEM-shSPC25 capable of crossing BBB. (A) Schematic of generation of AAV-pTMEM-shSPC25 and control AAV-pTMEM-scramble, using serotype PHP.B. The viral promoter also drives an RFP reporter connected with transgene with a p2A connector. (B) Transduction of mouse microglia cell line HMC3, mouse neuronal cell line HCN-2, mouse macrophage cell line RAW264.7, and mouse fibroblast cell line 3T3 with AAV-pTMEM-scramble. (C,D) Viral RFP was detected by RT-qPCR in different organs (C), or by gross image (D), 5 days after tail vein injection of AAV-pTMEM-scramble. (E,F) Transduction of HMC3 by AAV-pTMEMshSPC25 and control AAV-pTMEM-scramble. (E) Immunofluorescent staining for SPC25. (F) RT-qPCR for PSC25, iNOS and arginase. ${ }^{*} \mathrm{P}<0.05$. NS: non-significant. N=5. Scale bars are $20 \mu \mathrm{m}$. AAV, adeno-associated virus; BBB, blood-brain barrier; RFP, red fluorescent protein; RT-qPCR, quantitative reverse transcription polymerase chain reaction. 
expression of RFP reporter $5 \mathrm{~d}$ after infection (Figure 1B), which confirmed the specificity of TMEM119 promoter for microglia. We repeated these experiments in each cell line 5 times. The AAV-pTMEM-scramble was injected into mice and after $5 \mathrm{~d}$, RFP signals were exclusively detected in the mouse brain, and not in the mouse heart, skeletal muscle, stomach, liver, gallbladder, intestine, kidney, heart, or lung (Figure 1C), confirming both the capability of crossing BBB by AAV serotype PHP.B, and the specificity of TMEM119 promoter for microglia. This experiment was also repeated 5 times in each cell line. Moreover, RFP was only visualized in the brain region of the mice $5 \mathrm{~d}$ after tail vein injection of AAV-pTMEM-scramble (Figure 1D). Furthermore, in the AAV-pTMEM-shSPC25-transduced HMC cells, the levels of SPC25 were significantly reduced compared with the AAV-pTMEM-scramble-transduced HMC cells, by immunostaining for SPC25 (Figure 1E), and by RT-qPCR for SPC25 messenger RNA (mRNA) (Figure $1 F$ ). Interestingly, depletion of SPC25 did not alter polarization of microglia cell polarization in vitro, as the mRNA levels for iNOS and arginase in the transduced cells remained unchanged (Figure 1F). Here, 5 repeats were performed. Together, these data suggest that the generated AAV (serotype PHP.B)-pTMEM-shSPC25 is able to cross BBB and deplete SPC25 specifically in microglia.

\section{Administration with AAV-pTMEM-shSPC25 attenuates behavior disorder in AD-prone mice}

The APP/PS1 mice are AD-prone and typically develop obvious AD-associated behavioral and pathological disorders at 6 months of age. Thus, we performed single tail vein injection with AAV-pTMEM-shSPC25 or AAVpTMEM-scramble at 3 months of age, and waited for another 3 months before evaluating the mice. Each experimental group had 3 male and 3 female mice. In the Morris water-maze test for assessing spatial reference memory, the mice from the AAV-pTMEM-shSPC25treated and AAV-pTMEM-scramble-treated groups exhibited no difference in the path length in the visible platform phase (Figure $2 A$ ). However, the pTMEMshSPC25-treated mice used a significantly shorter path length in the hidden platform phase (Figure 2B) and took significantly shorter target quadrant occupancy (Figure 2C), compared to AAV-pTMEM-scramble-treated mice. For motor assessment, the pTMEM-shSPC25-treated mice required significantly a shorter time to cross a narrow beam (Figure 2D), and fell less frequently (Figure 2E), compared to AAV-pTMEM-scramble-treated mice. These data suggest that administration with AAV-pTMEM-shSPC25 attenuates behavior disorder in $\mathrm{AD}$-prone APP/PS1 mice.

\section{Administration with AAV-pTMEM-shSPC25 attenuates Aß deposition and p-Tau formation in APP/PS1 mouse brain}

To understand the underlying mechanisms, we first quantified the neuronal content in the CA1 region of mouse hippocampi at sacrifice. We detected significant attenuation in loss of neurons in AAV-pTMEM-shSPC25-treated mice, compared to AAV-pTMEM-scramble-treated mice (Figure $3 A$ ). Next, we analyzed the $\mathrm{A} \beta$ plaques in the CA1 region of mouse hippocampus. We detected a significantly smaller number of $A \beta$ plaques in the CA1 region of mouse hippocampus from AAV-pTMEM-shSPC25-treated mice, compared to AAV-pTMEM-scramble-treated mice, shown by representative images (Figure $3 B$ ), and by quantification (Figure $3 C$ ). The A $\beta$ levels were determined by ELISA, showing significantly reduced $A \beta$ levels in the CA1 region of the= hippocampi of AAV-pTMEM-shSPC25-treated mice, compared to AAV-pTMEM-scramble-treated mice (Figure 3D). Then, we analyzed p-Tau in the CA1 region of mouse hippocampi. We detected significantly less p-Tau formation in the CA1 region of mouse hippocampi from AAV-pTMEM-shSPC25-treated mice, compared to AAVpTMEM-scramble-treated mice, shown by quantification (Figure 3E) and representative images (Figure 3F). The p-Tau levels were determined by ELISA, showing significantly reduced p-Tau levels in the CA1 region of mouse hippocampi from AAV-pTMEM-shSPC25-treated mice, compared to AAV-pTMEM-scramble-treated mice (Figure 3G). Thus, administration with AAV-pTMEMshSPC25 attenuates $\mathrm{A} \beta$ deposition and p-Tau formation in APP/PS1 mouse brain.

\section{Administration with AAV-pTMEM-shSPC25 attenuates microglia outgrowth}

Finally, we examined the effects of AAV-pTMEMshSPC25 on microglia outgrowth in APP/PS1 mice. By immunostaining for a microglia-specific marker IBA-1 (16), we found that administration with AAV-pTMEM-shSPC25 massively reduced the number of microglia in the in the CA1 region of mouse hippocampi from AAV-pTMEM-shSPC25treated mice, compared to AAV-pTMEM-scrambletreated mice (Figure 4A). The CD11b-positive, CD45- 
A

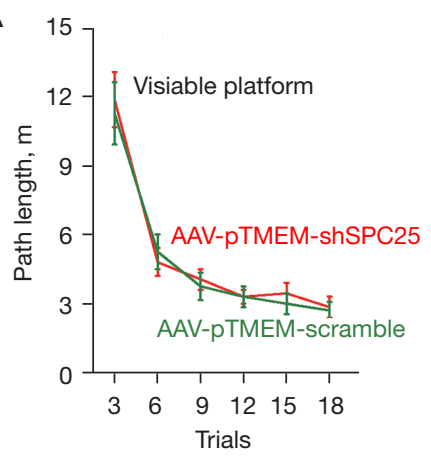

D

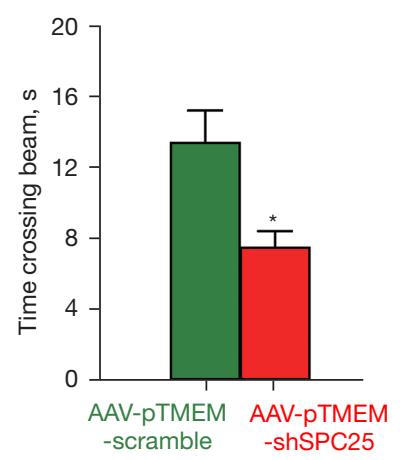

B

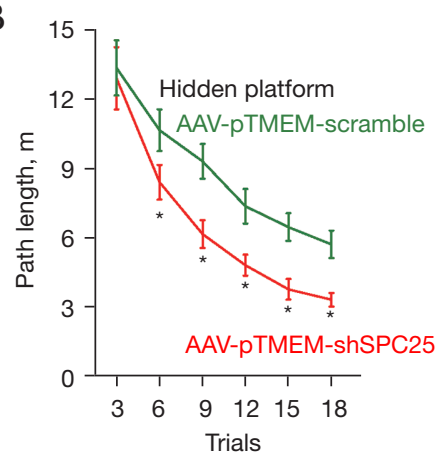

$\mathrm{E}$

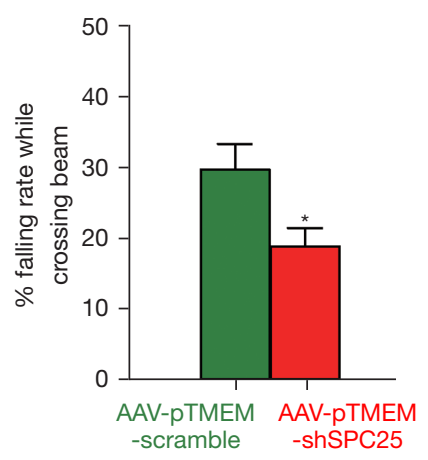

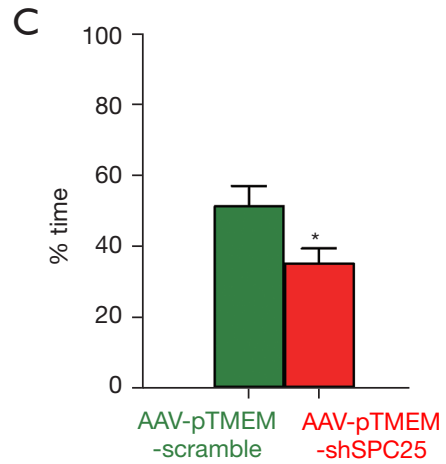

Figure 2 Administration with AAV-pTMEM-shSPC25 attenuates behavior disorder in AD-prone mice. We performed single tail vein injection with AAV-pTMEM-shSPC25 or AAV-pTMEM-scramble at 3 months of age, and waited for another 3 months before evaluating the mice. (A-C) A Morris Water Maze test for assessment of path length in the visible platform (A), path length in the hidden platform (B) and mean target quadrant occupancy (C). (D-E) A motor assessment test of time crossing a narrow beam (D) and falling rate during crossing the beam $(\mathrm{E}) .{ }^{*} \mathrm{P}<0.05 . \mathrm{N}=6$. AD, Alzheimer's disease; AAV, adeno-associated virus.

low microglia were isolated by flow cytometry (Figure 4B), showing significant reduction in percentage (Figure $4 C$ ) and total number (Figure $4 D$ ) in the in the CA1 region of mouse hippocampus from AAV-pTMEM-shSPC25-treated mice, compared to AAV-pTMEM-scramble-treated mice. Thus, the depletion of SPC25 may prevent AD progression through compromising microglia outgrowth.

\section{Discussion}

Abnormal mitosis is key feature of many diseases, especially tumor. Kinetochores are the major factors associated with normal and abnormal cell growth, through their regulation of mitotic chromosome segregation (6). The hetero-tetramer protein complex Ndc80 is made up of Ndc80, Nuf2, Spc24, and Spc25 to form the core of the kinetochore (7).

We have been studying the role of SPC25 in different diseases, which are highlighted by abnormal cell growth with aberrant expression of SPC25. For example, we have previously shown that SPC25 plays a non-redundant role in the proliferation and cell cycle progression of prostate cancer (8), and is the key factor to determine the stemness of prostate cancer cells (9). In response to a recent study on microglia profiling at single-cell level showing that microglia significantly upregulated SPC25 (10), we became inspired to examine whether this SPC25 upregulation in microglia may be necessary for microglia outgrowth and AD development.

In the current study, we studied the role of SPC25 in microglia and its effects on development of AD. Brain microglia are not only regarded as resident amyloid phagocytes, but also found to regulate many neuronal functions. Specially, chronic impairment in the interaction between microglia-neuron may lead to the permanence of synaptic and neuronal function failure exhibited in $\mathrm{AD}$ and Parkinson's diseases (2). In our approach, we prepared an AAV vector system that specifically depleted SPC25 in microglia. The selection of PHP.B serotype allowed 

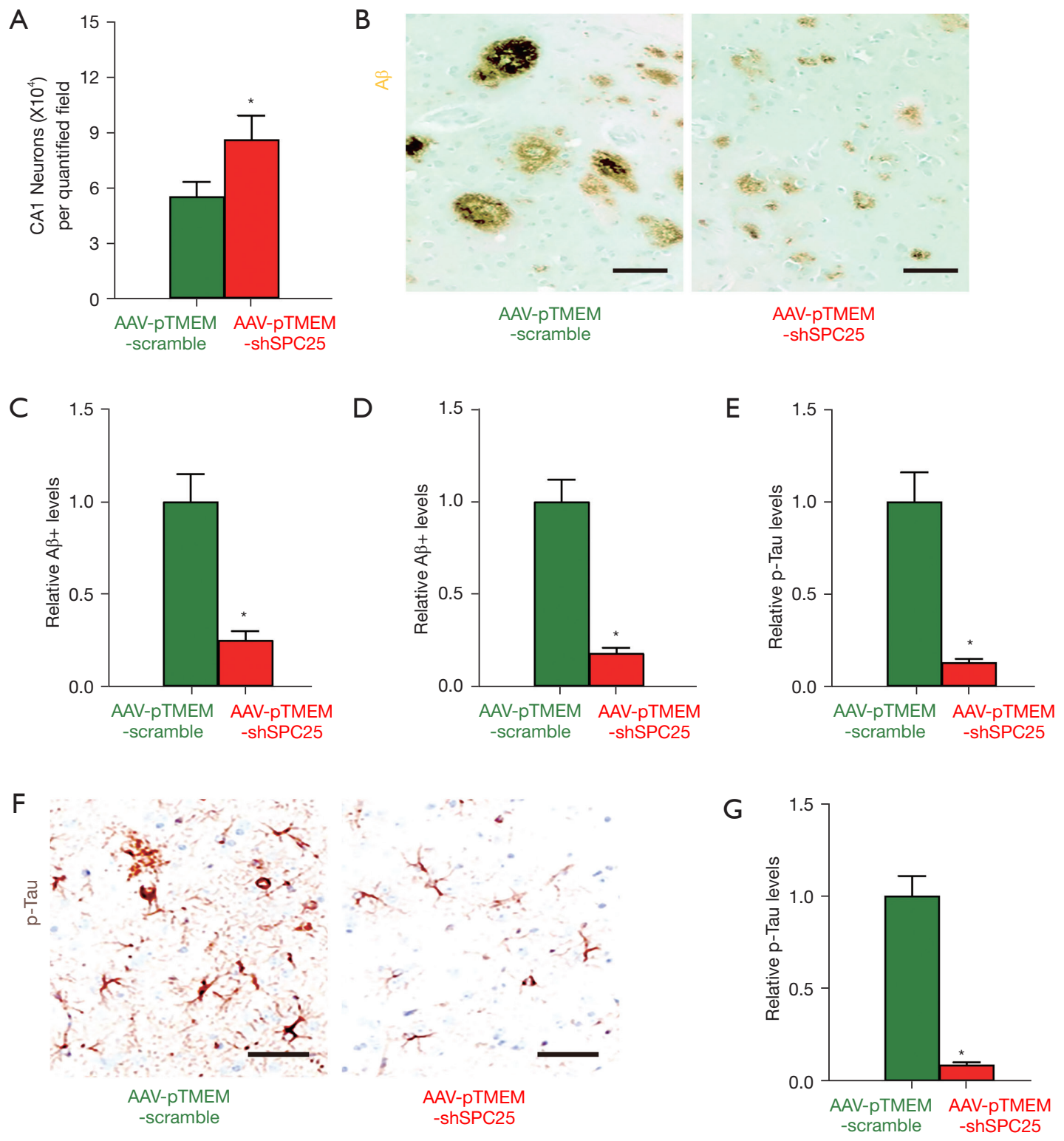

Figure 3 Administration with AAV-pTMEM-shSPC25 attenuates A $\beta$ deposition and p-Tau formation in APP/PS1 mouse brain. (A) Neuron loss in the CA1 region of mouse hippocampus. $(B, C)$ The A $\beta$ density in the CA1 region of mouse hippocampus was quantified at sacrifice, shown by representative images (B), and by quantification (C). (D) ELISA for A $\beta$ levels in the CA1 region of mouse hippocampus at sacrifice. (E,F) The p-Tau density in the CA1 region of mouse hippocampus was quantified at sacrifice, shown by quantification (E), and by representative images $(\mathrm{F}) .(\mathrm{G})$ ELISA for pTau levels in the CA1 region of mouse hippocampus at sacrifice. ${ }^{*} \mathrm{P}<0.05$. $\mathrm{N}=6$. Scale bars are $50 \mu \mathrm{m}$. AAV, adeno-associated virus; ELISA, enzyme-linked immunosorbent assay.

the virus to be administrated in the circulation and cross BBB (12-14), which was evidenced by absent expression of virus-specific reporter in non-brain organs. Moreover, we used a microglia-specific TMEM119 promoter to drive the shSPC25 or control scramble sequence (15). This promoter has been shown to be specific to microglia by many previous studies, and by us, since only microglia, but not macrophages, neurons, or fibroblasts were transduced to express the virus-specific reporter. These cell types are the major cell types in the brain. With these 2 strategies, we 

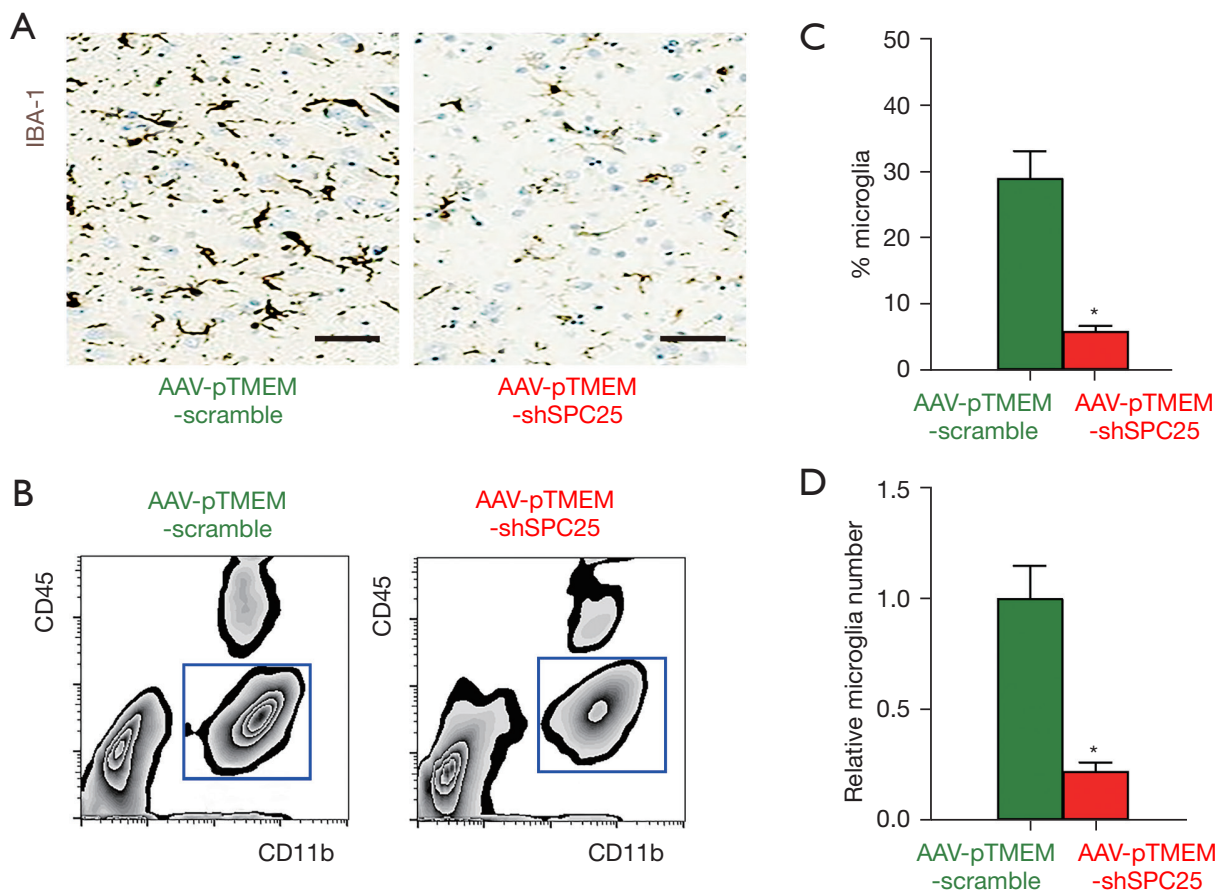

Figure 4 Administration with AAV-pTMEM-shSPC25 attenuates microglia outgrowth. (A) Immunostaining for a microglia-specific marker IBA-1 in the CA1 region of mouse hippocampus at sacrifice. (B) Representative flow charts for isolation of CD11b-positive, CD45-low microglia from the CA1 region of mouse hippocampus at sacrifice by flow cytometry. (C,D) Microglia percentage (C) and absolute number (D). ${ }^{*} \mathrm{P}<0.05$. Scale bars are $50 \mu \mathrm{m} . \mathrm{N}=6$. AAV, adeno-associated virus.

were able to deliver the shSPC25 specifically to microglia.

Our results showed significant reduction in microglia growth by shSPC25, and these effects on microglia growth appeared to dramatically reduce $\mathrm{AD}$ progression, which is consistent with a pivotal role of microglia in the pathogenesis of AD (3-5). Since recent studies have demonstrated a complex role of microglia in the development of neurodegenerative diseases (17), we also looked at the effect of SPC25 depletion on microglia phenotype. Depletion of SPC25 in microglia, however, did not alter the levels of iNOS and arginase, 2 markers for proinflammatory microglia and anti-inflammatory microglia, respectively (18), suggesting that the effects of SPC25 on microglia polarization is minimal. To summarize, in this study, by using a novel strategy, we revealed a clinic-translational method to interfere with $\mathrm{AD}$ progression through targeting microglia in vivo.

\section{Acknowledgments}

Funding: This work was supported by the Social Development Plan of Jiangsu Province-Standardization of key disease diagnosis and treatment project (BE2016715), Jiangsu Province Youth Medical Key Talent Program (QNRC2016457), Natural Science Foundation of Jiangsu Province (BK20191221), and Jiangsu Provincial Health Commission Project (H2017089).

\section{Footnote}

Reporting Checklist: The authors have completed the ARRIVE reporting checklist. Available at https://dx.doi. org/10.21037/atm-21-4064

Data Sharing Statement: Available at https://dx.doi. org/10.21037/atm-21-4064

Conflicts of Interest: All authors have completed the ICMJE uniform disclosure form (available at https://dx.doi. org/10.21037/atm-21-4064). The authors have no conflicts of interest to declare.

Ethical Statement: The authors are accountable for all aspects of the work in ensuring that questions related 
to the accuracy or integrity of any part of the work are appropriately investigated and resolved. Experiments were performed under a project license (SQK-20160037-Y) granted by institutional ethics board of the Affiliated People's Hospital of Jiangsu University, in compliance with institutional guidelines for the care and use of animals.

Open Access Statement: This is an Open Access article distributed in accordance with the Creative Commons Attribution-NonCommercial-NoDerivs 4.0 International License (CC BY-NC-ND 4.0), which permits the noncommercial replication and distribution of the article with the strict proviso that no changes or edits are made and the original work is properly cited (including links to both the formal publication through the relevant DOI and the license). See: https://creativecommons.org/licenses/by-nc-nd/4.0/.

\section{References}

1. van Kooten J, Binnekade TT, van der Wouden JC, et al. A Review of Pain Prevalence in Alzheimer's, Vascular, Frontotemporal and Lewy Body Dementias. Dement Geriatr Cogn Disord 2016;41:220-32.

2. Butovsky $\mathrm{O}$, Weiner HL. Microglial signatures and their role in health and disease. Nat Rev Neurosci 2018;19:622-35.

3. Sakae N, Heckman MG, Vargas ER, et al. Evaluation of Associations of Alzheimer's Disease Risk Variants that Are Highly Expressed in Microglia with Neuropathological Outcome Measures. J Alzheimers Dis 2019;70:659-66.

4. Fujikura M, Iwahara N, Hisahara S, et al. CD14 and Toll-Like Receptor 4 Promote Fibrillar A $\beta 42$ Uptake by Microglia Through A Clathrin-Mediated Pathway. J Alzheimers Dis 2019;68:323-37.

5. Strobel S, Grünblatt E, Heinsen H, et al. Astrocyteand Microglia-Specific Mitochondrial DNA Deletions Levels in Sporadic Alzheimer's Disease. J Alzheimers Dis 2019;67:149-57.

6. Tooley J, Stukenberg PT. The Ndc80 complex: integrating the kinetochore's many movements. Chromosome Res 2011;19:377-91.

7. Jeong J, Keum S, Kim D, et al. Spindle pole body component 25 homolog expressed by ECM stiffening is required for lung cancer cell proliferation. Biochem

Cite this article as: Cui F, Xu Z, Lv Y, Hu J. Role of spindle pole body component 25 in neurodegeneration. Ann Transl Med 2021;9(18):1432. doi: 10.21037/atm-21-4064
Biophys Res Commun 2018;500:937-43.

8. Cui F, Hu J, Fan Y, et al. Knockdown of spindle pole body component 25 homolog inhibits cell proliferation and cycle progression in prostate cancer. Oncol Lett 2018;15:5712-20.

9. Cui F, Tang H, Tan J, et al. Spindle pole body component 25 regulates stemness of prostate cancer cells. Aging (Albany NY) 2018;10:3273-82.

10. Mathys H, Adaikkan C, Gao F, et al. Temporal Tracking of Microglia Activation in Neurodegeneration at Single-Cell Resolution. Cell Rep 2017;21:366-80.

11. Jankowsky JL, Fadale DJ, Anderson J, et al. Mutant presenilins specifically elevate the levels of the 42 residue beta-amyloid peptide in vivo: evidence for augmentation of a 42-specific gamma secretase. Hum Mol Genet 2004;13:159-70.

12. Huang Q, Chan KY, Tobey IG, et al. Delivering genes across the blood-brain barrier: LY6A, a novel cellular receptor for AAV-PHP.B capsids. PLoS One 2019;14:e225206.

13. Matsuzaki Y, Tanaka M, Hakoda S, et al. Neurotropic Properties of AAV-PHP.B Are Shared among Diverse Inbred Strains of Mice. Mol Ther 2019;27:700-4.

14. Morabito G, Giannelli SG, Ordazzo G, et al. AAVPHP.B-Mediated Global-Scale Expression in the Mouse Nervous System Enables GBA1 Gene Therapy for Wide Protection from Synucleinopathy. Mol Ther 2017;25:2727-42.

15. Ruan C, Sun L, Kroshilina A, et al. A novel Tmem119tdTomato reporter mouse model for studying microglia in the central nervous system. Brain Behav Immun 2020;83:180-91.

16. Ahmed Z, Shaw G, Sharma VP, et al. Actin-binding proteins coronin-1a and IBA-1 are effective microglial markers for immunohistochemistry. J Histochem Cytochem 2007;55:687-700.

17. Jin MM, Wang F, Qi D, et al. A Critical Role of Autophagy in Regulating Microglia Polarization in Neurodegeneration. Front Aging Neurosci 2018;10:378.

18. Tsuda M. Microglia in the CNS and Neuropathic Pain. Adv Exp Med Biol 2018;1099:77-91.

(English Language Editor: J. Jones) 